\title{
A PROPERTY OF A CLASS OF NONLINEAR DIFFERENCE EQUATIONS
}

\author{
F. T. HOWARD
}

\begin{abstract}
Let $g(n)$ be a rational function of $n$ whose denominator is divisible by the same power of 2 for each $n$ and let $a_{1}$, $a_{2}, \cdots$ be any sequence of rational numbers such that for $n>1$, $a_{n}=g(n)\left(a_{1} a_{n-1}+a_{2} a_{n-2}+\cdots+a_{n-1} a_{1}\right)$. In this paper we determine the exact power of 2 dividing the denominator of $a_{n}$ for each $n$ and prove congruences $(\bmod 4)$ and $(\bmod 8)$.
\end{abstract}

1. Introduction. When dealing with special sequences of rational numbers, we often want to answer the following question: For a given prime $p$, what is the exact power of $p$ dividing the numerator or denominator of the $n$th number in the sequence? In general this is a difficult question to answer, especially, if nothing is known about the numbers other than a recurrence formula expressing the $n$th number of the sequence in terms of the previous numbers. Another generally difficult problem is to prove congruences for the numbers $\left(\bmod p^{k}\right), k \geqq 1$. For both of these problems it appears that the case $p=2$ is usually easier to deal with than $p>2$.

In this paper we consider any sequence $a_{1}, a_{2}, \cdots$ of rational numbers such that, for $n>1$,

$$
a_{n}=g(n) \sum_{k=1}^{n-1} a_{k} a_{n-k},
$$

where $g(n)$ is a rational function of $n$ which is divisible by exactly the same power of 2 for each $n$. Examples of such numbers are $a_{n}=B_{2 n} /(2 n)$ ! where $B_{2 n}$ is the $2 n$th Bernoulli number and $a_{n}=\left(\begin{array}{c}2 n-2 \\ n-1\end{array}\right) / n$. We shall determine the exact power of 2 dividing the denominator (or numerator if 2 does not divide the denominator) of $a_{n}$ and prove congruences $(\bmod 4)$ and $(\bmod 8)$. Examples are discussed in $\S 6$. We note that one of these examples is discussed in [4] and [3] and that a conjecture in [4] is proved in this paper.

Presented to the Society, November 20, 1971; received by the editors September 21, 1971.

AMS (MOS) subject classifications (1970). Primary 10A35, 10A40, 10A10.

Key words and phrases. Nonlinear difference equation, recurrence formula, rational function, congruence, Bernoulli number, Rayleigh function.

(c) American Mathematical Society 1973 
2. Preliminaries. The notation of the introduction and the definitions and lemmas of this section will be used in the subsequent sections.

Definition 2.1. Define $\theta(n)$ as the exponent of the highest power of 2 dividing the denominator of $a_{n}, n=1,2, \cdots$. If $\theta(n)$ is negative then $-\theta(n)$ is the exponent of the highest power of 2 dividing the numerator of $a_{n}$.

Definition 2.2. Define $t$ as the exponent of the highest power of 2 dividing the denominator of $g(n), n=2,3, \cdots$. If $t$ is negative then $-t$ is the exponent of the power of 2 dividing the numerator of $g(n)$.

Definition 2.3. Let $n$ be a positive integer and let $m$ be the number of nonzero terms in the base 2 expansion of $n$. Define $f(n, s)$ as the number of positive integers $r \leqq n / 2$ such that the number of nonzero terms in the base 2 expansion of $r$ plus the number of nonzero terms in the base expansion of $n-r$ is equal to $m+s$.

The following lemmas are proved in [3] and are necessary for the proofs of the congruences in this paper.

LEMMA 2.1. If there are $m$ nonzero terms in the base 2 expansion of $n$ then $f(n, 0)=2^{m-1}-1$.

LEMMA 2.2. Let $n=2^{e_{1}}+\cdots+2^{e_{m}}, m>1, e_{i}-e_{i+1}>1$, for $q$ terms $e_{i}, e_{i}-e_{i+1}=1$, for $m-q-1$ terms. Then $f(n, 1)=(q+1) 2^{m-2}$ if $e_{m} \geqq 1$ and $f(n, 1)=q 2^{m-2}$ if $e_{m}=0$.

LEMMA 2.3. Let $n=2^{e_{1}}+\cdots+2^{e_{m}}, e_{i}-e_{i+1}>s>0$, for $i=1, \cdots$, $m-1$ and $e_{m} \geqq s$. Then

$$
f(n, s)=\sum_{j=1}^{m}\left(\begin{array}{c}
m \\
j
\end{array}\right)\left(\begin{array}{c}
s-1 \\
j-1
\end{array}\right) \alpha(j) 2^{s+m-2 j-1},
$$

where $\alpha(j)=2$ if $s+m=2 j, \alpha(j)=1$ if $s+m>2 j$.

\section{The power of 2 dividing $a_{n}$.}

THEOREM 3.1. Let $n \geqq 1$ and let $m$ be the number of nonzero terms in the base 2 expansion of $n$. Then $\theta(n)=n \theta(1)+1+(n-1) t-m$.

Proof. The proof is by induction on $n$. The theorem is true for $n=1$. Assume it is true for $1, \cdots, n-1$ and let $x=n \theta(1)+1+(n-1) t-m$. We have

$$
2^{x} a_{n}=2^{x} g(n) \sum_{k=1}^{[n / 2]} \beta(k) a_{k} a_{n-k},
$$

where $\beta(k)=2$ if $k \neq n / 2, \beta(k)=1$ if $k=n / 2$. For any $k$ we consider $2^{x-t} \beta(k) a_{k} a_{n-k}$. Suppose there are $h$ nonzero terms in the base 2 expansion of $k$ and $w$ nonzero terms in the base 2 expansion of $n-k$. 
Case 1. $m \neq 1$. We have by our induction hypothesis

or

$$
2^{x-t} \beta(k) a_{k} a_{n-k}=2^{\theta(k)} a_{k} \cdot 2^{\theta(n-k)} a_{n-k} \cdot 2^{h+w-m}
$$

$$
=\left(2^{\theta(n / 2)} a_{n / 2}\right)^{2} \cdot 2^{m-1} \text { if } k=n / 2 .
$$

Hence every term on the right side of $(3.1)$ is congruent to $0(\bmod 2)$ except those for which $h+w=m$. By Lemma 2.1 there are $2^{m-1}-1$ such terms, an odd number.

Case 2. $m=1$. In this case every term on the right side of (3.1) is congruent to $0(\bmod 2)$ except when $k=n / 2$. Hence in both Cases 1 and 2 we have $2^{x} a_{n} \equiv 1(\bmod 2)$.

4. Congruences $(\bmod 4)$. We must assume that $a_{1}$ and $g(n)$ satisfy one of the following three sets of congruences for all $n$. We let $r$ stand for either 1 or 3 .

$$
\begin{aligned}
2^{t} g(n) & \equiv 2^{\theta(1)} a_{1} \equiv r(\bmod 4), & \\
2^{t} g(n) \equiv(-1)^{n} r(\bmod 4), & & 2^{\theta(1)} a_{1} \equiv-r(\bmod 4), \\
2^{t} g(n) \equiv(-1)^{n} r(\bmod 4), & & 2^{\theta(1)} a_{1} \equiv r(\bmod 4) .
\end{aligned}
$$

An example of (4.1) is $a_{1}=1, g(n)=1, n=2,3, \cdots$.

An example of (4.2) is $a_{1}=1 /(2 a+4), g(n)=2 /(a+2 n)$, where $a$ is odd.

An example of $(4.3)$ is $a_{1}=1 / 12, g(n)=-1 /(2 n+1)$.

THEOREM 4.1. Let $n=2^{e_{1}}+\cdots+2^{e_{m}}, e_{i}-e_{i+1}>1$, for $q$ terms $e_{i}$, $e_{i}-e_{i \dashv 1}=1$, for $m-1-q$ terms $e_{i}(i=1, \cdots, m-1)$. Then if (4.1) or (4.2) holds, we have, for $n>1$,

$$
2^{\theta(n)} a_{n} \equiv(-1)^{q} r(\bmod 4) .
$$

Proof. The proof is by induction on $n$. The theorem is true for $n=2$ since by equation (3.1) and Theorem 3.1

$$
2^{\theta(2)} a_{2} \equiv r\left(2^{\theta(1)} a_{1}\right)^{2} \equiv r(\bmod 4) .
$$

Assume the theorem is true for $2, \cdots, n-1$ and let $n$ satisfy the hypotheses of the theorem.

Case 1. $m=1$. In this case we have $n=2^{e_{1}}, e_{1}>0, q=0$ and by equation (3.1), Theorem 3.1 and our induction hypothesis we have

$$
2^{\theta(n)} a_{n} \equiv r\left(2^{\theta(n / 2)} a_{n / 2}\right)^{2} \equiv r(\bmod 4) .
$$

Case 2. $m=2$. In this case $n=2^{e_{1}}+2^{e_{2}}$ and we verify directly, as in Case 1, that the theorem holds in the four possible cases: ' $1-e_{2}>1, e_{2}>0$; $e_{1}-e_{2}>1, e_{2}=0 ; e_{1}-e_{2}=1, e_{2}>0 ; e_{1}-e_{2}=1, e_{2}=0$. 
The proofs in the final three cases are quite similar to each other. We shall state the cases and then prove Case 5.

Case 3. $m>2$ and in the base 2 expansion of $n$ there are two terms $2^{e_{i}}, 2^{e_{j}}$ such that $e_{i-1}-1>e_{i}>e_{i+1}+1$ and $e_{j-1}-1>e_{j}>e_{j+1}+1$. We note that $e_{i}$ could be either $e_{1}$ or $e_{m}$, in which case the conditions are $e_{1}>e_{2}+1$ or $e_{m-1}-1>e_{m}$.

Case 4. $\quad m>2$ and in the base 2 expansion of $n$ there is a term $2^{e_{i}}$ such that $e_{i}-e_{i+1}=1, e_{i-1}>1+e_{i}$ (if $\left.i \neq 1\right)$ and $e_{i+1}>1+e_{i+2}($ if $i+1 \neq m)$.

Case 5. $m>2$ and in the base 2 expansion of $n$ there is a term $2^{e_{i}}$ such that $e_{i}-e_{i+1}=1, e_{i+1}-e_{i+2}=1$ and $e_{i-1}-e_{i}>1$ (if $i \neq 1$ ).

To prove Case 5 we shall use the letters $h$ and $w$ as they were used in the proof of Theorem 3.1. We shall also assume that (4.1) holds, since the proof is very similar when (4.2) holds. We first want to consider those terms on the right side of equation (3.1) for which $h+w=m$. Let $E=$ $\left\{e_{1}, \cdots, e_{m}\right\}$. We know that if $h+w=m$ then the exponents of 2 in the base 2 expansions of $k$ and $n-k$ must be elements of $E$. Consider any distribution of the elements of $E-\left\{e_{i}, e_{i+1}\right\}$ between $k$ and $n-k$ for which there is at least one element assigned to $k$ and at least one element assigned to $n-k$. Then there are four possibilities for the assignments of $e_{i}$ and $e_{i+1}$ and we have $2^{\theta(k)} a_{k} \cdot 2^{\theta(n-k)} a_{n-k}$ is congruent to $(-1)^{z}, z=1$ or -1 if $e_{i}, e_{i+1}$ and $e_{i+2}$ are all assigned together; $(-1)^{z+1}$ if $e_{i+1}$ and $e_{i+2}$ are assigned together, $e_{i}$ assigned differently; $(-1)^{z+1}$ if $e_{i}$ and $e_{i+1}$ are assigned together, $e_{i+2}$ assigned differently; $(-1)^{z+2}$ if $e_{i}$ and $e_{i+2}$ are assigned together, $e_{i+1}$ assigned differently.

Notice that the sum of all such terms $2^{\theta(k)} a_{k} \cdot 2^{\theta(n-k)} a_{n-k}$ is congruent to $0(\bmod 4)$.

If all the elements of $E-\left\{e_{i}, e_{i+1}\right\}$ are assigned to $k$ (or $n-k$ ) then by our induction hypothesis $2^{\theta(k)} a_{k} \cdot 2^{\theta(n-k)} a_{n-k}$ is congruent to $(-1)^{q}$ if $e_{i}$ and $e_{i+1}$ are assigned together (and hence separated from $\left.e_{i+2}\right) ;(-1)^{q}$ if $e_{i+1}$ is assigned with $e_{i+2}$ and $e_{i}$ is assigned differently; $(-1)^{q+1}$ if $e_{i}$ is assigned with $e_{i+2}$ and $e_{i+1}$ is assigned differently.

Therefore we have

$$
\begin{aligned}
2^{\theta(n)} a_{n} & \equiv r\left[2(-1)^{q}+(-1)^{q+1}\right]+2 r f(n, 1)(\bmod 4) \\
& \equiv(-1)^{q} r(\bmod 4)
\end{aligned}
$$

since, by Lemma $2.2, f(n, 1) \equiv 0(\bmod 2)$.

The next theorem is proved in a similar way. We omit the proof.

THEOREM 4.2. Let $n=2^{e_{1}}+\cdots+2^{e_{m}}, e_{i}-e_{i+1}>1$, for $q$ terms $e_{i}$, $e_{i}-e_{i+1}=1$, for $m-1-q$ terms $e_{i}(i=1, \cdots, m-1)$. Then if (4.3) holds, we have, for $n>1$,

$$
2^{\theta(n)} a_{n} \equiv(-1)^{q+n} r(\bmod 4)
$$


5. Congruences $(\bmod 8)$. In this section we must assume that $a_{1}$ and $g(n)$ satisfy one of the following three sets of congruences, where $r$ is either $1,3,5$ or 7 .

$$
\begin{array}{rlrl}
2^{t} g(n) & \equiv 2^{\theta(1)} a_{1} \equiv r(\bmod 8), & \\
2^{t} g(n) \equiv(-1)^{n} r(\bmod 8), & & 2^{\theta(1)} a_{1} \equiv-r(\bmod 8), \\
2^{t} g(n) & \equiv(-1)^{n} r(\bmod 8), & & 2^{\theta(1)} a_{1} \equiv r(\bmod 8) .
\end{array}
$$

We shall also make use of Lemma 2.3, Theorem 3.1, Theorem 4.1 and Theorem 4.2.

THEOREM 5.1. Suppose any one of (5.1), (5.2) or (5.3) holds for all $n$. If $n=2^{e_{1}}+\cdots+2^{e_{m}}, e_{i}-e_{i+1}>2$, for $i=1, \cdots, m-1$ and $e_{m} \geqq 2$, then

$$
\begin{aligned}
2^{\theta(n)} a_{n} & \equiv 7 r(\bmod 8) \quad \text { if } m \text { is even, } \\
& \equiv 5 r(\bmod 8) \quad \text { if } m \text { is odd. }
\end{aligned}
$$

Proof. The proof is by induction on $m$. We first verify that it is true for $m=1,2,3$. If $n=2^{e_{1}}$, then

$$
2^{\theta(n)} a_{n} \equiv r f(n, 1)+4 r f(n, 2) \equiv 5 r(\bmod 8) .
$$

If $n=2^{e_{1}}+2^{e_{2}}$, then

$$
2^{\theta(n)} a_{n} \equiv r f(n, 0)+6 r f(n, 1)+4 r(f(n, 2)-1)+2 r \equiv 7 r(\bmod 8) .
$$

If $n=2^{e_{1}}+2^{e_{2}}+2^{e_{3}}$, then

$$
2^{\theta(n)} a_{n} \equiv 3 r f(n, 0)+2 r f(n, 1)+4 r f(n, 2)+4 r \equiv 5 r(\bmod 8) .
$$

Assume the theorem is true for $1,2,3, \cdots, m-1$ and $n=2^{e_{1}}+\cdots+2^{e_{m}}$. If $m$ is even we have

$$
2^{\theta(n)} a_{n} \equiv r f(n, 0)+6 r f(n, 1)+4 r f(n, 2) \equiv 7 r(\bmod 8) .
$$

If $m$ is odd, we have

$$
2^{\theta(n)} a_{n} \equiv 3 r f(n, 0)+2 r f(n, 1)+4 r f(n, 2) \equiv 5 r(\bmod 8) .
$$

\section{Examples.}

EXAMPLE 6.1. Let $a_{n}=\left(\begin{array}{c}2 n-2 \\ n-1\end{array}\right) / n$. Then $a_{1}=1$ and (see [6, pp. 74-75])

$$
a_{n}=\sum_{k=1}^{n-1} a_{k} a_{n-k} .
$$

If $n=2^{e_{1}}+\cdots+2^{e_{m}}, e_{i}-e_{i+1}>1$, for $q$ terms $e_{i}$, then, by Theorem 3.1, $\theta(n)=1-m$. Therefore the exponent of the highest power of 2 dividing 
$\left(\begin{array}{c}2 n-2 \\ n-1\end{array}\right)$ is $m+s-1$, where $s$ is the exponent of the highest power of 2 dividing $n$. Furthermore,

$$
2^{1-m-s}\left(\begin{array}{c}
2 n-2 \\
n-1
\end{array}\right) \equiv 2^{-s} n(-1)^{a}(\bmod 4) .
$$

If $e_{i}-e_{i+1}>2, e_{m} \geqq 2$, then

$$
\begin{aligned}
2^{1-m-s}\left(\begin{array}{c}
2 n-2 \\
n-1
\end{array}\right) & \equiv 2^{-s} 7 n(\bmod 8), \quad \text { if } m \text { is even, } \\
& \equiv 2^{-s} 5 n(\bmod 8), \quad \text { if } m \text { is odd. }
\end{aligned}
$$

We note that Wolstenholme in about 1880 pointed out that the highest power of 2 dividing $\left(\begin{array}{c}2 n-1 \\ n\end{array}\right)$ is $m-s-1$ where $m$ is the number of nonzero terms in the base 2 expansion of $2 n-1$ and $s$ is the exponent of the highest power of 2 dividing $n$. Cesaro, Kummer, Van den Broeck and others considered this type of problem also [2, pp. 270-272].

EXAMPLE 6.2. Let $a_{n}=B_{2 n} /(2 n)$ ! where $B_{2 n}$ is the $2 n$th Bernoulli number, defined by

$$
\frac{x}{e^{x}-1}=\sum_{r=0}^{\infty} B_{r} \frac{x^{r}}{r !}
$$

Then $a_{1}=1 / 12$ and (see [7, p. 146])

$$
a_{n}=\frac{-1}{2 n+1} \sum_{k=1}^{n-1} a_{k} a_{n-k}
$$

If $n=2^{e_{1}}+\cdots+2^{e_{m}}, e_{i}-e_{i+1}>1$, for $q$ terms $e_{i}$, then we have $\theta(n)=$ $1+2 n-m$. Since $2 n-m$ is the exponent of the highest power of 2 dividing $(2 n)$ ! [2, p. 263] we have the well-known result that if $n \geqq 1$, the denominator of $B_{2 n}$ is divisible by 2 but not by 4 . Also, by Theorem 4.2,

$$
2^{1+2 n-m} B_{2 n} /(2 n) ! \equiv(-1)^{a+n+1}(\bmod 4) .
$$

We note that in [1] it is pointed out that

$$
2 B_{2 n} \equiv 1(\bmod 4), \quad n>1,
$$

and in fact

$$
2 B_{2 n} \equiv 1+4 n(\bmod 16), \quad n>2 .
$$

Thus it follows that, for $n>1$,

$$
2^{m-2 n}(2 n) ! \equiv(-1)^{a+n+1}(\bmod 4) .
$$


EXAMPLE 6.3. Let $a_{n}=\sigma_{2 n}(a / b)$, the Rayleigh function of argument $a / b, a$ odd, $b$ even (see [5]). Then $a_{1}=b / 4(a+b)$ and

$$
a_{n}=\frac{b}{a+b n} \sum_{k=1}^{n-1} a_{k} a_{n-k} .
$$

This example is discussed in [4] and [3]. We note that Theorem 4.1 proves a conjecture in [4], namely that if $b=(2 k+1) 2^{t}, t>0$, then

$$
2^{\theta(n)} \sigma_{2 n}(a / b) \equiv(-1)^{q}((2 k+1) / a)(\bmod 4) .
$$

\section{REFERENCES}

1. L. Carlitz, A property of the Bernoulli numbers, Amer. Math. Monthly 67 (1960), 1011-1012.

2. L. E. Dickson, History of the theory of numbers. Vol. 1, Publication No. 256, Carnegie Institution of Washington, Washington, D.C., 1919.

3. F. T. Howard, A combinatorial problem and congruences for the Rayleigh function, Proc. Amer. Math. Soc. 26 (1970), 574-578. MR 42 \#1756.

4. - A property of the Rayleigh function, Proc. Amer. Math. Soc. 24 (1970), 719-723. MR 40 \#7495.

5. N. Kishore, The Rayleigh function, Proc. Amer. Math. Soc. 14 (1963), 527-533. MR 27 \#1633.

6. C. L. Liu, Introduction to combinatorial mathematics, McGraw-Hill, New York, 1968. MR 38 \#3154.

7. N. E. Norlund, Vorlesungen über Differenzrechnung, Springer-Verlag, Berlin, 1924.

Department of Mathematics, Wake Forest University, Winston-Salem, North Carolina 27109 